\title{
Leer el arte o cómo la lectura nos enseña pintura
}

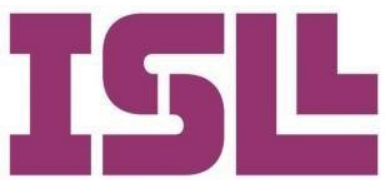

Número 11

Junio de 2019

\author{
Enric Falguera Garcia \\ Universidad de Lleida \\ https://orcid.org/0000-0001-6021-2169
}

Recibido: $15-01-2019$

Aceptado: 01-05-2019

Pág. 74 a la 83 Resumen:

Palabras clave:

Competencia lectora,

Arte, Poesía,

Metacognición, Álbum

ilustrado.
El presente artículo es una propuesta de comprensión del texto poético porque análisis de la competencia lectora a posibilita descubrir los recursos literarios partir de cinco textos sobre el mundo que se esconden tras la creación poética. del arte. El artículo es una Este hecho, además, pone de relieve como aproximación al análisis de textos que la lectura sobre el mundo del arte hablan de pintura con un doble incrementa el desarrollo de la creatividad objetivo: mejorar la competencia y la imaginación, tanto desde el punto de lectora y los conocimientos artísticos vista pictórico como poético.

de los lectores. El planteamiento del El artículo analiza, por una parte, cuatro artículo es comprobar como la lectura álbumes ilustrados sobre pintores e de libros sobre pintores, movimientos historia del arte para ejemplificar como su artísticos, épocas pictóricas nos dota de lectura facilita al lector ser consciente de los conocimientos previos necesarios los procesos de comprensión del texto y para posteriormente poder contemplar del cuadro; y por otra, poemas sobre un cuadro, admirarlo y entenderlo. pinturas del poeta catalán Agelet i Garriga Leer arte nos enseña arte y que ponen de relieve los mecanismos de la paralelamente leer una pintura genera creación poética y, en consecuencia, de la unos mecanismos cognitivos, parejos a lectura poética.

las estrategias de lectura que se generan cuando leemos un texto escrito. Leer sobre arte nos dota de los conocimientos técnicos que posibilitan la lectura de la pintura y su comprensión contextualizada.

Por otro lado, cuando leemos poesía basada en una obra pictórica, no solo esta ayuda a entender mejor el cuadro, sino que analizar los procesos creativos que ha desencadenado el poeta para trasladar al papel las sensaciones de la pintura, permite una mejor lectura y 


\section{Introducción. Leer el arte}

Cuando hablamos de leer, pensamos en la lectura de textos escritos, sean de la tipología que sea, formales como una noticia o informales como un WhatsApp o un tuit. La moda y expansión de los álbumes ilustrados ha facilitado la ampliación del concepto de lectura a la imagen. Ahora leer es también contemplar una ilustración, interpretar su significado, valorar la técnica ilustrativa, la utilización del color, dominar el lenguaje audiovisual y sus nociones teóricas como el plano medio o plano cenital, establecer relaciones entre imagen y texto se ha convertido en una nueva forma de lectura. Así, un álbum ilustrado, en el que el texto y la imagen se interrelacionan para construir un significado coherente y cohesionado que permite al lector / espectador interpretar e inferir unos hechos, entender una narración y adquirir una conciencia crítica a partir de la conjunción entre dicho texto e su imagen con el objetivo de consolidar y desarrollar su competencia literaria, nos acerca a la lectura de imágenes. (Arañó, 1994; Duran, 2010; Tabernero, 2009, 2012, 2013; Bosch, 2007). No es el único caso. El lenguaje publicitario también se sirve de la imagen como herramienta de lectura, de inferencia de un mensaje que acompaña al texto.

\section{Objetivos e hipótesis}

Así, pues, partimos de la hipótesis que leer un libro y mirar una pintura desarrolla procesos y actitudes similares. Implica una relación entre el lector/espectador y la obra observada. Sin ir más lejos la conocida definición de lectura que propone (Solé, 1992, p. 21): "Leer es un proceso de interacción entre un lector y un texto, proceso según el cual el primero intenta satisfacer, obtener una información pertinente para los objetivos que guían su lectura" puede utilizarse también para definir la lectura de la imagen, de la ilustración y particularmente de la pintura, del arte en general, como una experiencia de aprendizaje, que contribuye en el proceso de formación de individuos receptivos, críticos, dialogantes, imaginativos y reflexivos (Eisner, 2004). Contemplar arte, leer un cuadro implica un tipo de experiencia que es producto de la metacognición (Eisner, 2002) porque los procesos mentales activados son los mismos que los exigidos para la lectura de textos escritos. Contemplar una pintura es interactuar con ella, es satisfacer un objetivo, sea informativo, estético, histórico, o puramente placentero. $\mathrm{Y}$ en esa interacción es donde se produce el aprendizaje, las conexiones metacognitivas que nos permiten leer el cuadro, inferir su significado o describirlo literalmente (Deleuze y Guattari, 1987).

De hecho, las relaciones entre literatura y arte han centrado el interés de los principales artistas y pensadores de todos los tiempos y han sido objeto de debate desde la antigüedad. Es Lessing quien en su libro Laocoon marca las relaciones entre las dos artes. Así, a pesar de considerar que ambas tienen como objetivo la imitación (mejorada) de la naturaleza, Lessing cree que "la sucesión temporal es el ámbito propio del poeta, mientras que la sucesión espacial lo es del pintor" (Lessing, 2014, p. 36). Es decir: que el espacio de la pintura está limitado a la esfera de lo visible, mientras que el de la literatura es más vasto porque abarca tanto lo visible como lo invisible.

A la luz de esta nueva forma de lectura, parece evidente que el discente lector debe aprender nuevas maneras de lectura y una de ellas es saber leer un cuadro (Laneyrie-Dagen, 2013). Para ello necesita de los conocimientos previos necesarios: "Conocer las técnicas de composición, movimiento, iluminación, color, temática y simbología de las obras. En definitiva, conocer la Historia del Arte y sus protagonistas y, especialmente, conocer muy bien el entorno cultural, 
político, económico, religioso y social donde se desarrolla la vida y la actividad del artista, porque si no es así, podremos ver su pintura, pero no entender el mensaje que está transmitiéndonos" (Martín-Gil, 2011, pp. 26-27).

Llegados a este punto, la lectura del cuadro nos lleva de nuevo a la lectura del texto. Para interpretar el cuadro debemos (in)formarnos sobre él y esa información la encontramos en los textos que hablan de arte. Se trata, pues, de un camino de ida y vuelta. Leer una obra de arte es leer un texto que nos habla de ella, de su entorno, de su historia, de su creación. Y dicho acercamiento al arte, a la pintura a través de los textos, se puede producir, básicamente, de tres formas distintas, a partir de tres objetivos:

1. leer textos de no ficción que nos acerquen a la historia del arte, a sus técnicas pictóricas, estilos o épocas.

2. leer textos de ficción que nos contextualicen el pintor, su época, su estilo, su visión del mudo y el arte.

3. leer textos, especialmente poéticos, que ellos mismos sean textos pictóricos, como pinturas que reproducen otras pinturas. No es trata simplemente de describir una pintura, sino de ir más allá, trasladando al texto la técnica del cuadro y sus motivos en un tipo de poesía denominada écfrasis.

\section{Método de análisis: aprender a leer, aprender a mirar}

\section{Leer para aprender}

Actualmente, parece imprescindible el uso de la lectura como herramienta de acceso al conocimiento (Clar, Llauradó, Riera, Quinquer y Roca, 2007; Vicente-Yagüe, 2016). De hecho, los currículos escolares insisten en que la competencia lectora es un elemento fundamental para el desarrollo de las competencias básicas y un instrumento primordial para vehicular todo tipo de aprendizajes. Así, el trabajo de comprensión lectora en el aula pierde su sentido si no tiene un objetivo claro integrado dentro de una tarea. Las áreas no lingüísticas constituyen, por tanto, excelentes contextos de uso real de la lectura porque permiten al discente integrar nueva información y establecer una red de aprendizajes que le permite revisar, generar, y confrontar todo tipo de conocimientos de manera personal y colectiva (Solé, 1992). Así, para saber sobre arte hay que leer sobre arte. Pero leer sobre arte requiere de unos conocimientos previos necesarios sobre las características intrínsecas del lenguaje pictórico y sobre las estructuras textuales de los textos artísticos. Aprender a leer en las áreas no lingüísticas es, por tanto, aprender a acceder a los contenidos del área, y las actividades de comprensión de textos didácticos son actividades de aprendizaje tanto de lengua como del área trabajada. Es decir: si leemos textos sobre el mundo del arte, aprenderemos a leer, aprenderemos lengua, pero, también, aprenderemos a interpretar un cuadro, a leer un estilo pictórico o un pintor y para hacerlo deberemos conocer las características lingüísticas y textuales del arte: el volumen, la profundidad y la perspectiva, el uso y significación del color y la iluminación, los estilos y periodos artísticos, los temas, la composición y el movimiento o el lenguaje iconológico.

Aprender arte se ha convertido en los últimos años en un objetivo fundamental de la educación infantil y primaria (Bamford, 2009). La apuesta por una educación sensible a las necesidades y al proceso de desarrollo de la infancia ha favorecido el desarrollo de una educación 
artística que lo conecte con su realidad, a partir de una forma de educar basada en la empatía, en la identificación de uno mismo y en la proyección hacia el otro (Morin, 1999, p. 52). Tal y como señala (Mendívil Trelles de Peña, 2011, p. 28) "Privar al niño de la experiencia artística es privarlo de un modo de aprehensión de su realidad". Esta apuesta por conectar la infancia con su realidad a través del arte se fundamenta en la exploración de dos habilidades básicas: la experimentación y la imaginación (Eisner, 2004). La experimentación artística permite la posterior elaboración de conceptos y conocimientos y la elaboración de una sensorialidad que conecta la infancia con su mundo (Vasquez, 2010), a la vez que le abre un mundo imaginario: "Despertar la imaginación es una forma de encontrar nuevos sentidos y significados, es crear imágenes de realidades alternativas, abrirse a otras posibilidades diferentes a las conocidas, inventar visiones de nuestro mundo, pero sobre todo imaginar es refinar y usar la sensibilidad" (Mendívil Trelles de Peña, 2011, pp. 28-29) y el arte potencia y desarrolla esta imaginación que nos permite conocernos mejor y conocer nuestra realidad.

De entre la abundante bibliografía sobre libros de arte dirigidos a niños, subrayamos tres álbumes ilustrados: El abc del arte para niños de Amanada Renshaw y Gilda Williams, Las tijeras de Matisse de Jeanette Winter y El sonido de los colores de Barb Rosenberg. Destacamos estos libros porque el primero se ha convertido en un manual imprescindible para acercarse a la historia de la pintura y los libros de Renshaw y Winter devienen dos magníficos ejemplos de cómo acercarse a la obra de un pintor a través de la sensibilidad y la multisensorialidad.

El abc del arte para niños es un paseo por la historia del arte, especialmente del contemporáneo. De los 30 autores y obras que presenta más de la mitad, diecisiete, son obras contemporáneas. El libro no propone conocimientos sobre la biografía del pintor, su estilo o su época, sino que se acerca a la obra que analiza desde un punto de vista original. Por ejemplo, en el momento de hablar de "Boda campesina" de Pieter Bruegel el viejo plantea el cuadro como una gran fiesta y la autora propone dos preguntas: “¿Puedes oír el ruido que hay en este cuadro?, ¿Crees que hace frio en esta sala? Las preguntas realizadas desde la multisensorialidad facilitan el acercamiento de los niños a la obra maestra y les permite iniciar un proceso de entendimiento del cuadro desde la reflexión cognitiva. El arte nos hace preguntas que debemos contestar o no. Lo importante es que nos permita establecer conexiones con nuestro mundo, que nos permita comprenderlo (López-Rodríguez, 2007, p. 32). En numerosos casos, estas reflexiones y este proceso metacognitivo se traslada a una actividad práctica. En "Las cuatro estaciones" de Arcimboldo, la autora propone componer una cara a partir del método del pintor italiano. Es decir construir una cara con los elementos que la identifican. Como vemos la lectura del cuadro se plantea como un juego. Lo mismo ocurre con "Mujer llorando" de Pablo Ruiz Picasso. La autora se acerca al cuadro desde la emoción, focalizando en el hecho de llorar y cuáles son las expresiones que tomamos cuando 1loramos. A partir de aquí plantea una nueva cuestión sobre los motivos que han hecho llorar a la mujer e inicia el proceso cognitivo de observación y comprensión.

Una de las peculiaridades de este volumen es que no solo se acerca a la pintura, sino que también analiza obras poco canónicas en el mundo clásico del arte, como la fotografía, el mobiliario, las perfomance o las actuaciones artísticas como los envoltorios de Christo.

Por su lado, el libro de Jeanette Winter, Las tijeras de Matisse, se aproxima a un periodo concreto de la obra de artista francés: sus recortes de papel pintado, de su última 
etapa creativa. Se trata, pues, de un recurso creativo próximo al alumnado que puede imitar la obra del pintor francés. El leitmotif del álbum ilustrado es el deseo, la voluntad inquebrantable de Matisse por pintar, por crear pese a las obligaciones familiares o a la enfermedad: "Era feliz y hacía feliz a la gente con sus pinturas" (Winter, 2013, p. 8). Precisamente la incapacidad de pintar, le lleva a utilizar las tijeras como método de creación, como forma de expresar sus pensamientos y sentimientos. Es precisamente este el objetivo del volumen: fomentar la libre expresión a través de la creación artística entre los lectores del álbum. El Matisse postrado en la cama, vive a través de los recortes de papel pintado, goza de la vida a través del arte que lo hace todo posible. Incluso hasta la genial metáfora final del libro: " $\mathrm{Y}$ Y si alguna de las estrellas que vemos en el cielo ha salido de las tijeras de Matisse?" (Winter, 2013, p. 28). Así, el álbum se convierte en una herramienta para reflexionar sobre las relaciones entre arte y creatividad y una manera de acercar didácticamente la creación artística a las aulas. (Arañó Gisbert, 1994).

En la misma línea de superación personal, el tercer volumen analizado se aproxima a la obra del pintor ruso: Vasily Kandinsky. El sonido de los colores de Barb Rosenstock profundiza en un aspecto de la psicología del personaje central: la sinestesia. Kandinsky padecía, al parecer, una alteración genética que le permití percibir los colores como sonidos y los sonidos como colores. Esta particularidad queda reflejada en su obra, pionera del arte abstracto. Como veremos, más tarde, la sinestesia es un recurso literario fundamental para transformar la pintura en texto poético. Kandinsky también transforma los sentidos en su arte. El álbum ilustrado es un buen ejemplo para explicar la sensibilidad artística de los niños, porque muestra cómo se pueden utilizar los sentidos para hacer arte y, una vez más, enseña la voluntad artística del protagonista que contra la opinión familiar y de sus profesores busca su propio camino creativo. No se limita a imitar el arte figurativo y expresa su mundo sensitivo en la abstracción.

Ambos álbumes ilustrados usan la narración para ejemplificar los mundos creativos de los autores mostrados. En el caso de Matisse el onirismo y la abstracción de los recortes de papel pintado se manifiesta en el uso de un lenguaje repleto de metáforas: "sus ojos estaban llenos de tristeza", "las tijeras son unos instrumentos maravillosos", "las caras veían sus sueños" (Winter, 2013). Observamos que se trata de metáforas cercanas precisamente a la sinestesia. En el caso de Kandinsky, el texto es mucho más extenso, descriptivo y técnico. Utiliza numerosos referentes semánticos de la pintura, tales como colores o técnicas pictóricas. Aun así, aparecen claras sinestesias como "sus pinturas que gritaban”, "oía cantar los colores, veía bailar la música" o "los atronadores arcos de aguamarina" (Rosenstock, 2015).

Finalmente, el álbum ilustrado de Rosenstock termina con una pregunta: “¿Cómo te hace sentir? Una vez más, la lectura del cuadro, del mundo del pintor se dirige hacía su lector, su espectador para interrogarle sobre sus sentimientos, sus emociones en un dialogo entre razón y placer y estética (López-Rodríguez, 2007).

Por el contrario, otra forma de acercarse al mundo del arte a través de la lectura es a partir de textos narrativos de ficción que tratan sobre un periodo o sobre un artista. Este es el caso de $E l$ misterio Velázquez de Eliacer Cansino (1998), premio Lazarrillo 1997. 
La novela está protagonizada por Nicolás Pertusato el enano retratado en "Las meninas". La trama nos presenta a Nicolás Pertusato, un niño que es arrancado del seno familiar para que sirva en la Casa Real. Él mismo nos cuenta su viaje, su llegada a la corte y su posterior trabajo en palacio. Poco a poco irá conociendo a todo el mundo e irá involucrándose en las intrigas de la corte. Por una de ellas conoce a Diego de Velázquez y termina siendo uno de los protagonistas del futuro cuadro de las Meninas. El joven ayudará a Velázquez a terminar su cuadro y le ayudará a seguir adelante con su proyecto en una aventura misteriosa y algo mágica que nos ayuda al lector a realizar hipótesis sobre algunos de los misterios que envuelven el cuadro del pintor sevillano. En especial el misterio sobre la cruz de Santiago.

La novela transmite de forma excelente el contexto histórico y la lucha del pintor por la defensa de su arte, acercándose así el lector a una visión historicista y contextualizadora de la historia del cuadro.

\section{La pintura poética. Écfrasis}

Pero si hay una verdadera manera de leer arte, esta es leer textos literarios que reproducen fielmente el estilo y las sensaciones de la pintura por la utilización que hacen de los recursos literarios y porque su modelo de inspiración se encuentra en la obra pictórica. Se trata de un tipo de poesía denominada écfrasis que muchos escritores han abordado a lo largo de los años. Para el presente artículo nos centraremos en la obra de un poeta catalán del siglo XX: Jaume Agelet i Garriga (1888-1981) que a lo largo de su producción poética, escribe poemas basados en el arte pictórico. Sus poemas reproducen la temática, el estilo y el ambiente del cuadro o el pintor objeto de la inspiración del poema y para conseguirlo utiliza unos recursos muy concretos: la imagen y la comparación, los verbos y adjetivos de movimiento y la sinestesia. Veámoslo con algunos ejemplos concretos.

Agelet tiene varios poemas dedicados a temas directamente relacionados con la pintura. Algunos de ellos, y que analizamos aquí, son: "En clase de dibujo", "Bodegón", "Colores", "Ante un cuadro de Henri Matisse", "Esbozo", "Mirando una pintura de Jansen", y "Momento de Delft". Así en este último poema, que reproducimos:

El agua en el canal es densa de verdor; se mecen unas barcas de neblina.

Ahora un cisne repliega su claridad;

blanquea el muelle silencio una gaviota.

Una calle avanza, largo de soledad

y junto al agua amarillo, marchito, tiembla.

El frío, extraviado,

por los callejones, palpando los muros, rueda.

El poeta describe un paisaje de manera muy plástica, casi impresionista. Probablemente, esta descripción sea fruto de la contemplación del cuadro del pintor holandés Johannes Vermeer: "Vista de Delft". En el poema se describen todos los elementos del cuadro, y se observa un elemento fundamental: la luz. Una luz sólo intuida, en penumbra, gris, gracias a la presencia de la neblina, el verde denso, casi oscuro, del canal, el cisne que repliega su claridad y el frío. El color también está presente gracias a la adjetivación, pero también a los verbos (blanquea), los 
sustantivos que rápidamente quedan asociados a un color (la gaviota y el cisne, blancos), el amarillo del agua y el gris del frío y la niebla. El juego de contrastes lumínicos del cuadro se pone de manifiesto en el poema. Además, Agelet salva la tradicional inamovilidad de la pintura con los verbos de movimiento y el uso de imágenes y personificaciones: "Una calle avanza", "Frio descarriado", "El frío rueda", "Se mecen unas barcas".

Estos recursos también entran en acción en otros poemas. En el poema "Bodegón" la adjetivación contribuye a dar movimiento a la naturaleza muerta: "Se apilan cerezas palpitantes" o "como unas rebanadas de pan sangriento". Además, utiliza verbos de movimiento para objetos inanimados "cestas que se decantan", y el recurso más sorprendente de todos: la incorporación de un elemento inexistente en cualquier naturaleza muerta pictórica pero que contribuye desde su ausencia a movilizar la escena: "guardan oscuras, invisibles manos". La personificación del cuadro se produce con la aparición de las manos que, como invisibles, todo el mundo sabe que no se encuentran en la pintura, pero dan volumen y proyección vital en la cesta de cerezas y, pues, al cuadro. Así, Agelet a través de la cuidadosa descripción nos ofrece la pintura en todas sus dimensiones de color y de espacio. De hecho, Agelet utiliza a menudo adjetivos que dan la impresión de que pueden tocar los objetos que califican, como ocurre con estas manos invisibles que hacen presente la naturaleza muerta (Falguera, 2010). Con estos poemas el lector aprende como el uso de los recursos literarios, metáforas e imágenes, pero también el uso del sentido figurado y la lexicalización de palabras o la formación de nuevos significados denotativos, permite enriquecer, crear nuevo lenguaje, nuevas significaciones que nos acercan a la comprensión del mundo y a la formación lectora y cultural. De esta forma, la lectura de estos poemas permite al joven lector construir técnicamente un poema pictórico, y en realidad un poema cualquiera, porque el uso de las metáforas, personificaciones y sinestesias se encuentran en la base de toda construcción poética. Descodificar este tipo de poemas ayuda al lector a comprender los mecanismos de la creación poética $\mathrm{y}$, de rebote a comprender la técnica constructiva de una pintura, a leer un cuadro.

Como hemos ido manifestando, la sinestesia se convierte en un recuso cabal para la lectura del arte. Esta consiste en atribuir la calidad del objeto principal a conceptos e ideas que están ligadas. Así, por ejemplo, en "Las gaviotas vuelan blancas, / suavemente azules de mar", el azul del mar ha sido atribuida a las gaviotas. A veces el objeto principal no está expresado y lo tenemos que buscar a través precisamente del color: "Pino vacilante de voz oscura, / envuelto de un aliento azul" donde el aliento azul es el cielo gracias a la asociación del color: cielo azul. De hecho, la sinestesia sirve para luchar contra la tradicional inmovilidad propia de la pintura y la poesía ecfrástica. El intercambio de las sensaciones produce en el lector una impresión de dinamismo y la luz y el color, como hemos visto, tienen un papel clave. Un buen ejemplo de poema sinestésico "Colores". Un poema donde estos se asocian a los diferentes elementos de la naturaleza: el verde en la zarza, el rojo a la cereza, el amarillo al sol, el aliento azul que es el cielo, el ojal sangriento de la fuente viva es agua, el blanco es la espuma viva de crin o el atardecer (con un nuevo juego de luces) es la claridad áurea que yace. Otro ejemplo es el poema "A clase de dibujo". Este poema es uno de los pocos textos ageletians que no tienen aparentemente movimiento y nos lo presenta como un cuadro estático. El poema es la descripción de diferentes elementos naturales: la luna, el sol, la ciruela, la lluvia y el viento, pero también de estados anímicos, como el gozo. Así, se asocia unos con otros a través de los colores. La descripción de la naturaleza en este poema como en la anterior evoca también un tono franciscano, de amor por las cosas sencillas. Hay, sin embargo, en el texto un hecho significativo: así como la mayoría de los elementos descritos pueden dibujarse, 
los elementos centrales del poema la lluvia y el viento, que aglutinan todos los colores, son de difícil representación pictórica, además, son también centrales para que reúnen el conjunto de la naturaleza en habitar el bosque. Finalmente, el poema termina con la llanura, una reflexión metaartística que, desierta y blanca como el papel del poeta o la tela del pintor, permite (re)crear el paisaje a través del lápiz de colores o las palabras, ambos elementos, un vez más, de un mismo arte. $\mathrm{Y}$ es que Agelet no concibe pintura y poesía como dos artes diferentes que establecen un diálogo entre ellas, sino como una sola arte. No escribe poemas sobre pintura sino que todos sus poemas son pinturas para la utilización que hace del color y las sensaciones y sentimientos vinculados al mismo.

Cuando la creación remite al objeto recreado como sucede en el poema "Ante un cuadro de Henri Matisse", entonces el texto reproduce la sensación de indeterminación de la pintura, el trazo impresionista y el poema se convierte en poco preciso a través del léxico utilizado: "Brilla tibia la escama" (en lo que es un nuevo ejemplo de sinestesia), "es blancura que escurre", "luz blanca que se deshace", y, finalmente, el pescado que derrama en la plata. Ahora, los colores van languideciendo, se hacen imprecisos para reproducir la obra de Matisse. Lo mismo sucede en el poema "Mirando una pintura de Jansen" donde el estilo difuminado del pintor francés se traslada al poema: "Un mundo, todo lento, / que se va fundiendo / y desnudando, silente".

Agelet es pues un caso excepcional entre los poetas que devienen pintores porque hace de sus poemas pinturas y nos descubre los mecanismos de construcción de ambas artes.

\section{Conclusiones}

Hemos comprobado como la lectura y divulgación de los álbumes ilustrados ha ampliado el concepto tradicional de leer a la lectura de imágenes, de ilustraciones y a partir de estas, es posible la lectura de imágenes que remiten al mundo de la historia del arte. Los jóvenes disponen de múltiples formas de acercarse al mundo del arte a través de la lectura y como esta deviene una herramienta imprescindible para descubrir y profundizar sobre conceptos y características propias del mundo pictórico. Pero también hemos visto como leer sobre pintura, y especialmente leer poesía que reproduce la pintura, nos descubre y enseña mecanismos propios del proceso lector.

Leer sobre arte nos permite conocer mejor una realidad que nos identifica como humanos, como seres sensibles (Eisner, 2004) y nos dota de unos conocimientos que nos permiten gozar de un cuadro, de una pintura y nos adentran en un placer estético, que nos posibilita desarrollar la imaginación y la expresividad. En este sentido obras como las analizadas nos facilitan aprender sobre el arte, una de las formas de expresión más genuinamente humanas.

Por otro lado, leer textos que reproducen las cualidades de la pintura nos posibilita comprobar como el lenguaje artístico presenta un gran nexo de unión y nos permite ver como los recursos propios de la literatura, el ritmo, el verso, la rima, los recursos literarios, el uso denotativo del lenguaje, la capacidad de asociar realidades diversas sirven para expresar un sentimiento, una emoción que también se da en un cuadro. Leer pintura ecfrástica es aprender a leer literatura porque sus mecanismos de creación se fundamentan justamente en la utilización de aquellos recursos que se asemejan a la pintura, que la convierten en arte, que identifican e individualizan el lenguaje literario. Leer poesía sobre pintura es aprender a interpretar la poesía, sus mecanismos y leer textos expositivos sobre pintura es aprender a contemplar la pintura, a preciar sus 
características intrínsecas. Ut pictura poesis como afirmaba Horacio, la pintura como la poesía, porque leer ambas es ampliar los horizontes de expectativas del lector, es aprender, es en definitiva ser mejores seres humanos.

\section{Referencias}

Agelet i Garriga, J (2008). Tria de versos. Lleida: Publicaciones de la Universitat de Lleida.

Arañó Gisbert, J.C. (1994). Arte, educación y creatividad. Pixel-Bit: Revista de medios y educación, 2, 65-87.

Bamford, A. (2009). El factor ¡Wuau! El papel de las artes en la educación. Un estudio internacional sobre el impacto de las artes en la educación. Barcelona: Octaedro.

Bosch, E. (2007) Hacia una definición de álbum. Anuario de Investigación en literatura infantil y juvenil, 5, 25-46.

Cansino, E (1998). El misterio Velázquez. Madrid: Bruño.

Clar, R., Llaurado, A., Riera, A., Quinquer, D. y Roca, M. (2007) Hablar, leer y escribir para aprender desde las áreas curriculares. Aula de Innovación Educativa, 159, 7-9

Deleuze, G. y Guattari, F. (1987). Mil mesetas, capitalismo y esquizofrenia. Valencia: Pre-Textos.

Duran, T. (2010). L'àlbum dins la narrativa visual. Articles de didàctica de la llengua i literatura, $52,10-22$.

Eisner, E. (2002) El arte y la creación de la mente. Barcelona: Paidós.

Eisner, E. (2004) El Arte y la Creación de la Mente: El Papel de Las Artes Visuales en la Transformación de la Conciencia. Madrid: Paidos

Falguera, E. (2010) La pintura d'un poeta, Actes del XVè Col·loqui Internacional de Llengua i Literatura Catalanes. Barcelona: PAM.

Laneyrie-Dagen, N. (2013). Leer la pintura. Barcelona: Larousse.

Lessing, G. E. (2014). Laocoonte o sobre los límites de la pintura y la poesía. Barcelona: Herder Editorial.

López-Rodríguez, F. (2007) (ed.). La educación artística en la escuela. Barcelona: Graó.

Martín-Gil, F. (2011). Claves para entender un cuadro. Madrid: AIIM

Mendívil Trelles de Peña, L. (2011). El arte en la educación de la primera infancia: una necesidad impostergable. Educación, Vol. XX, 39, 23-36

Morin, E. (1999). Los siete saberes necesarios para la educación del futuro. México: Unesco.

Renshaw, A. (2006). El abc del arte para niños. Barcelona: Phaidon.

Rosenstock, B. (2015). El sonido de los colores. Barcelona: Juventud.

Solé, I. (1992). Estrategias de lectura. Barcelona: Graó.

Tabernero, R. (2013). Claves para una poética de la recepción del libro-álbum: Un lector inserto en una "inmensa minoría. [online]. IBBY. http://www.ibby.org/index.php?id=1062

Tabernero, R. (2012). La hipertextualidad como fundamento de construcción en la literatura "iluminada": la formación del lector en el libro-álbum y en el libro ilustrado. Leer Hipertexto. Del marco hipertextual a la formación de lector literario. Barcelona: Octaedro.

Tabernero, R. (2009). Leer mirando. El libro-álbum en la promoción de hábitos lectores. Claves para una poética de su lectura. I Encuentro Internacional de Estudio y Debate. Literatura Infantil y Matices. p. 9-44.

Vasquez, C. (2010). El arte un derecho humano. Recovered from http://www.chalenavasquez.com/pdf/El_arte,_un_derecho_humano.pdf 
Vicente-Yagüe, M. ${ }^{a}$ I. (2016). Artes y literatura en la LOMCE: mecanismos para la comprensión de lecturas plurales. Investigaciones sobre lectura, 5, 59-69.

Winter, J (2013). Las tijeras de Matisse. Barcelona: Juventud. 


\section{Read art or how reading teaches us painting}

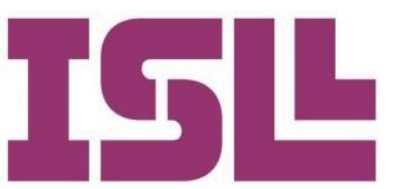

Number 11

2019 June

\section{Enric Falguera Garcia}

Universidad de Lleida

https://orcid.org/0000-0001-6021-2169
Received: 15-01-2019

Accepted: 01-05-2019

Pag. 84 to 92

\section{Keywords}

Reading cometence, Art, Poetry, Metacognition, Ilustrated album.

\section{Abstract:}

This article is a proposal for an analysis the painting to the paper, which allows a of reading competence based on five better reading and understanding of the texts about the art world. It presents an poetic text because it enables the approach to the analysis of texts that discovery of the literary resources that speak of painting with a double hide behind the poetic creation. This fact objective: to improve the reading also highlights how reading about the art competence and artistic knowledge of world enhances the development of readers. The approach of the article is creativity and imagination, both pictorial to verify how reading texts about and poetic.

painters, artistic movements, and The article analyzes three artistic epochs gives us the preliminary illustrated albums on picturesque and art knowledge necessary to contemplate, history and a novel, and shows how they admire, and understand a painting. makes the reader aware of the processes of Reading about art teaches us art and, in understanding the text and the picture. It parallel, how to read a painting then examines a series of poems about generated by cognitive mechanisms, paintings by the Catalan poet Agelet i similar to the reading strategies that are Garriga that highlight the mechanisms of in place when we read a written text. poetic creation and, consequently, of Reading about art gives us the poetic reading. technical knowledge that makes possible the reading of the painting and its contextualized understanding.

On the other hand, reading poetry based on a pictorial work not only helps to understand the picture more, but it also involves an analysis of the creative processes the poet has triggered to transfer the sensations of 


\section{Introduction. Reading the art}

When we talk about reading, we think about reading written texts, whether they be formal (such as items of news) or otherwise (e.g., WhatsApp messages or tweets). Illustrated albums have become fashionable, and they have increased in popularity. This has facilitated the extension of the concept of reading to the image. Reading now includes contemplating an illustration, interpreting its meaning, valuing the illustrative technique (such as the use of color), mastering the audiovisual language and its theoretical notions such as the middle plane or zenith plane, and establishing relationships between image and text. Thus, an illustrated album, in which the text and the image interconnect to build a coherent meaning that permits the reader / spectator to interpret and infer facts, understand a narrative, and acquire a critical awareness through the conjunction between the text and its image with the objective of consolidating and developing its literary competence, brings us closer to reading images. (Arañó, 1994; Bosch, 2007; Duran, 2010; Tabernero, 2009, 2012, 2013). Illustrated albums are not the only example. Advertising language also uses the image as a reading instrument, inferring a message that accompanies the text.

\section{Goals and hypothesis}

Thus, we start from the hypothesis that reading a book and looking at a painting cultivate similar processes and attitudes. It implies a relationship between the reader/spectator and the work observed. Without going any further, the well-known definition of reading as "a process of interaction between a reader and a text, the process according to which the former tries to satisfy, obtaining information relevant to the objectives guide their reading" (Solé, 1992, p. 21) can also be used to define the reading of the image, of the illustration, and particularly of the painting and of art in general, as a learning experience, which contributes to the process of formation of receptive, critical, dialoguing individuals, imaginative and reflective (Eisner, 2004). Contemplating art, or reading a painting, implies a type of experience that is a product of metacognition (Eisner, 2002) because the activated mental processes are the same as those required for reading written texts. To contemplate a painting is to interact with it and to satisfy an objective, whether it be informational, aesthetic, historical, or purely pleasant; and it is in that interaction where learning occurs, in the metacognitive connections that allow us to read the picture, infer its meaning, or describe it literally (Deleuze \& Guattari, 1987).

In fact, relations between literature and art have attracted the interest of leading artists and thinkers since ancient times. In Laocoon, Lessing marked the relationships between the two arts. Thus, despite considering that both have as their objective the (improved) imitation of nature, Lessing believed that "the temporal succession is the poet's own domain, while the spatial succession is the painter's." (Lessing, 2014, 36) In other words, the space of the painting is limited to the sphere of the visible, while that of literature is more vast because it encompasses both the visible and the invisible.

In the light of this new way of reading, it seems clear that the reader must learn new techniques. One is knowing how to read a picture (Laneyrie-Dagen, 2013). For this, they need necessary prior knowledge: "To know the techniques of composition, movement, lighting, color, theme and symbology of the works. In short, to know the History of Art and its protagonists and, 
especially, to know very well the cultural, political, economic, religious and social environment where the artist's life and activity takes place, because if not, we can see his painting but not understanding the message that is being transmitted to us" (Martín-Gil, 2011, pp. 26-27).

At this point, reading the art leads us back to reading the text. To interpret the picture we must (en) train ourselves in it and the information found in texts that talk about art. It is therefore a to-and-fro process. Reading a work of art is reading a text that tells us about it, its surroundings, its history, and its creation. This approach to art, to painting through texts, can be produced, basically, in three different ways, based on three objectives:

1. Read nonfiction texts that bring us closer to the history of art, its pictorial techniques, styles, or epochs.

2. Read fiction texts that contextualize the painter, his time, his style, his vision of mute and art.

3. Read texts, especially poetic, that themselves are pictorial texts, for instance paintings that reproduce other paintings. It is not simply a question of describing a painting, but of going further, translating the technique of the painting and its motifs into a type of poetry called ecphrasis.

\section{Method of analysis: learn to read, learn to look}

\section{Reading to learn}

Currently, it seems essential to use reading as an instrument of access to knowledge (Clar et al., 2007; Vicente-Yagüe, 2016). In fact, school curricula insist that reading proficiency is a fundamental element for the development of basic skills and a primary requirement for all kinds of learning. Thus, the work of reading comprehension in the classroom loses its meaning if it does not have a clear objective integrated into a task. Therefore, non-linguistic areas constitute excellent contexts for the real use of reading because they allow students to integrate new information and establish a learning network that allows them to review, generate, and confront all kinds of knowledge in a personal and collective way (Solé, 1992); so, to know about art you have to read about art. But reading about art requires some necessary prior knowledge about the intrinsic characteristics of pictorial language and about the textual structures of artistic texts. Learning to read in non-linguistic areas is, therefore, learning to access the contents of the area, and the activities of understanding didactic texts are learning activities both in the language and in the area worked. That is to say, if we read texts about the art world, we will learn to read and we will learn language, but, also, we will learn to interpret a painting, and to read a pictorial style or a painter, and to do so we must know the linguistic and textual characteristics of art: volume, depth, and perspective, the use and significance of color and lighting, artistic styles and periods, themes, composition and movement, and iconological language.

As has been noted, learning art has become in recent years a fundamental objective of preschool and primary education (Bamford, 2009). The commitment to an education sensitive to the needs and the process of childhood development has favored the development of an artistic education that connects it to childhood using empathy and the identification of oneself with the other (Morin, 1999, p. 52). As Mendívil Trelles de Peña (2011) pointed out, "To deprive the child 
of the artistic experience is to deprive him of a way of apprehension of his reality" (p. 28). This commitment to connect childhood with its reality through art is based on the exploration of two basic skills: experimentation and imagination (Eisner, 2004). Artistic experimentation allows the subsequent elaboration of concepts and knowledge and the development of a sensory apparatus that connects childhood with its world (Vasquez, 2010), while opening an imaginary one: "Awakening the imagination is a way to find new senses and meanings, to create images of alternative realities, to open up to other possibilities different from those known, to invent visions of our world, but above all to refine and use sensitivity" (Mendívil Trelles de Peña, 2011, pp. 28-29). Art enhances and develops this imagination that allows us to know ourselves better and to know our reality.

Amongst the abundant bibliography on art books aimed at children, we highlight three illustrated albums: The ABC of Art for Children by Amanda Renshaw and Gilda Williams, Matisse's Scissors by Jeanette Winter, and The Sound of Colors by Barb Rosenstock. The ABC of Art for Children has become an essential manual to approach the history of painting. It walks the reader through art history, especially contemporary art, with sensitivity and multisensorially. Of the 30 authors and works presented, seventeen are contemporary. The book does not present knowledge about the painter's biography, their style or their time, but rather Renshaw and Williams approach the works they analyze from an original point of view. For example, when talking about "Peasant Wedding" by Pieter Bruegel the old man raises the picture at a big party, and the authors propose two questions: "Can you hear the noise in this picture? Do you think it's cold in this room?" The questions asked from a multisensory angle ease the approach of children to the masterpiece, allowing them to begin the process of understanding the picture using cognitive reflection. Art asks us questions that must be answered or not. The important thing is that it allows the observer to establish connections with their world, and to understand it (LópezRodríguez, 2007, p. 32). In many cases, these reflections and this metacognitive process moves the reader towards a practical activity. For Arcimboldo's “The Four Seasons," the authors propose that the reader composes a face using the method of the Italian painter, that is, to build a face with the elements that identify it. Thus, the reading of the picture is presented as a game. The same goes for "Woman Crying" by Pablo Ruiz Picasso. The authors approach the painting using emotion, focusing on the fact of crying and the expressions that are formed when we cry. A question is asked about the reasons that have made the woman cry, and this starts the cognitive process of observation and understanding. One of the particularities of this volume is that it not only discusses painting, but also analyzes small canonical works, photography, furniture, and artistic performances such as Christo's wrappings.

Jeanette Winter's book, Matisse's Scissors, looks at a specific period of the French artist's work - his last creative stage- using his wallpaper clippings. It is, therefore, a creative resource for students to imitate his work. The leitmotif is Matisse's desire, the unwavering will to paint, to create, despite family obligations or illness: "He was happy and made people happy with his paintings" (Winter, 2013, p. 8). It was precisely the inability to paint that led him to use scissors as a method of creation, as a means to express his thoughts and feelings. This is precisely the objective of the volume: to promote free expression through artistic creation. The bedridden Matisse lives through wallpaper clippings, enjoys life through an art that makes everything possible - and the final metaphor of the book is couched within a question: "What if some of the stars we see in the sky have come out of Matisse's scissors?" (p. 28). Thus, the album becomes 
an instrument to reflect on the relationship between art and creativity, and to introduce artistic creation into the classroom (Spider Gisbert, 1994).

Along the same lines of self-improvement, the third volume presents the work of the Russian painter Vasily Kandinsky. The sound of Barb Rosenstock's colors delves into one aspect of the central character's psychology: synesthesia. Kandinsky apparently suffered from a genetic alteration that allowed him to perceive colors as sounds and sounds as colors. This particularity is reflected in his pioneering abstract work. As we will see later, synesthesia is a fundamental literary resource for transforming painting into poetic text. Kandinsky also transforms the senses into his art. The illustrated album is an effective way to explain artistic sensibility to children, because it shows how the senses can be used to make art and, once again, it teaches the artistic will of the protagonist who seeks his family's opinion and his teacher's own creative path. Kandinsky is not limited to imitating figurative art, and he expresses his sensitive world in abstraction.

Both Winter and Rosenstock use narration to illuminate the artists' creative worlds. In Matisse's case, the dreamlike nature and abstraction of wallpaper clippings are manifested in the use of language full of metaphors. Thus, "his eyes were full of sadness," "the scissors are wonderful instruments," and "the faces saw their dreams" (Winter, 2013). We observe that these are metaphors that approximate to synesthesia. In Rosenstock's book, the text is much more extensive, descriptive, and technical. It uses numerous semantic references, for example to colors or pictorial techniques. Synesthesia appears in phrases such as "his screaming paintings," "I heard the colors sing, I watched the music dance," and "the thundering aquamarine arches" (Rosenstock, 2015).

Finally, Rosenstock's illustrated album ends with a question: "How does it make you feel?" Once again, the reading of the painter's world is directed towards the reader, his spectator, through questions about his emotions, in a dialogue between reason and pleasure and aesthetics (López-Rodríguez, 2007).

Another way of reading art is through fictional narratives that deal with a particular period or an individual artist. One example is El misterio Velázquez [The Velázquez Mystery] by Eliacer Cansino (1998), which won the 1997 Lazarrillo Prize.

The novel features Nicolás Pertusato, the dwarf who is portrayed in "Las Meninas." He is a child who is torn from his family to serve in the Royal House. He tells us of his journey, his arrival at the court, and his subsequent work in the palace. Little by little, he gets to know everyone and becomes involved in the intrigues of the court. He meets Diego de Velázquez and ends up being one of the protagonists in the future Meninas painting. The young man helps Velázquez to finish his painting and to move forward with his project in a mysterious and somewhat magical adventure that helps the reader to hypothesise about some of the mysteries that surround the Sevillian's painting, especially with regard to the mystery surrounding the cross of Santiago. The novel conveys brilliantly the historical context of the work and the painter's struggle to defend his art. 
But if there is a true way to read art, it is to read literary texts that faithfully reproduce the style and sensations of paintings through literary resources and use as their inspirational model the work itself. An example of this approach is a form of poetry known as ecphrasis. This article will now focus on the work of a twentieth-century Catalan poet, Jaume Agelet i Garriga (1888-1981) who, throughout his poetic oeuvre, wrote poems based on pictorial art. The poems reproduce the theme, style and atmosphere of the painting or focus on the painter who is the inspiration for the poem. Agelet uses very specific resources: image and comparison, verbs and adjectives of movement, and synesthesia. Some concrete examples are now provided.

Agelet has several poems dedicated to subjects directly related to painting. Some of them, which are to be analyzed herein, include "In Drawing Class", "Still Life", "Colors", "Before a Painting by Henri Matisse", "Sketch", "Looking at a Painting by Jansen", and "Moment of Delft"
El agua en el canal es densa de verdor; se mecen unas barcas de neblina.
Ahora un cisne repliega su claridad;
blanquea el muelle silencio una gaviota.
Una calle avanza, largo de soledad
y junto al agua amarillo, marchito, tiembla.
El frío, extraviado,
por los callejones, palpando los muros, rueda.

In this latter poem, Agelet describes a landscape in a very plastic, almost impressionistic way. This description is probably the result of the contemplation of the painting by the Dutch painter Johannes Vermeer, "View of Delft". In the poem all the elements of the painting are described, and a fundamental element is observed: the light. A light only intuited in gloom, and gray, thanks to the presence of the mist, the dense, almost dark green of the canal, the swan that replicates its clarity, and the cold. The color is presented through adjectivation and verbs (blanquea), and the nouns that are quickly associated with a color (the seagull and the swan, white), the yellow of the water, and the gray of the cold and the fog. The play of lighting contrasts in the painting is revealed in the poem. In addition, Agelet compensates for the traditional stillness of painting with the verbs of movement and the use of images and personifications: "A street advances", "Cold wayward", "The cold wheel" and "Boats are rocked".

These resources also come into play in other poems. In "Bodegón" the adjective helps to give movement to the still life: "Throbbing cherries are stacked" or "like a few slices of bloody bread". In addition, the poet uses motion verbs for inanimate objects, for example "baskets that decant" and the most surprising resource of all: the incorporation of an element that does not exist in any pictorial still life but contributes in its absence to mobilize the scene: "they keep dark, invisible hands." The personification of the painting occurs through the appearance of the hands that everyone knows are not in the painting; but that give volume to the cherry basket and project it, and therefore, the painting. Thus, Agelet, through such careful description, offers us the painting in all its dimensions of color and space. In fact, he often uses adjectives that give the impression that the objects in the painting can be touched, as is the case with the invisible hands 
that give life to still life (Falguera, 2010).

With these poems the reader learns how the use of literary resources, metaphors and images, but also the use of figurative meaning and the lexicalization of words or the formation of new denotative meanings enriches the reader and enables them to create new language, new meanings that bring us closer to the understanding of the world and to the reading and cultural formation of the object. In this way, the reading of these poems allows the reader to construct technically a pictorial poem, and in reality an ordinary poem, because the use of metaphors, personifications, and synesthesia are at the base of all poetic construction. Decoding these types of poems helps the reader to understand the mechanisms of poetic creation and, returning to understand the construction technique of a painting, to read a painting.

As has been mentioned, synesthesia becomes an abundant resource for the reading of art. It consists of attributing the quality of the main object to concepts and ideas that are linked. Thus, for example, in "Seagulls fly white, / soft blue of sea" the blue of the sea has been attributed to the seagulls. Sometimes the main object is not expressed, and we have to look for it through color: "Wavering pine with a dark voice, / wrapped in a blue breath" where the blue breath is the sky thanks to the association of color: blue sky. In fact, synesthesia serves to counter the traditional immobility of painting and ecphrastic poetry. The exchange of sensations produces in the reader an impression of dynamism and light and color, as has been seen, they have a key role. A good example of a synesthetic poem is "Colors". Here the colors are associated with the different elements of nature: the green of the bush, the red of the cherry, the yellow of the sun, the blue breath that is the sky, the bloody eyelet of the living fountain that is water, the white that is the live froth of mane, or the sunset (using a new play of lights) with its the golden clarity. Another example is "A Drawing Class". This poem is one of the few ageletian texts that apparently have no movement; the painting is presented as a static picture. The poem describes different natural elements: the moon, the sun, the plum, the rain, and the wind, and also moods, such as joy. Thus, it associates each through colors. The description of nature in this poem as in the previous one also evokes a Franciscan tone, of love of simple things. There is, however, a significant fact in the text: just as most of the elements described can be drawn, the central elements of the poem -the rain and the wind- which bring together all the colors are difficult to paint, but they are also central in bringing together the whole of nature to inhabit the forest. Finally, the poem ends with the plain, a metaartistic reflection that, deserted and as white as the role of the poet or the painter's fabric, allows the (re)creation of the landscape through the colored pencil or words, both elements, once more, of the same art. Agelet does not conceive painting and poetry as two different arts that establish a dialogue, but as a single art. He does not write poems about painting, but all his poems are paintings through his use of color and the sensations and feelings linked to it.

When the creation refers to the object recreated, as happens in the poem "Before a Painting by Henri Matisse", then the text reproduces the feeling of indeterminacy of the painting and the impressionist strokes, and the poem paints an imprecision through lexical devices: "The scale shines warm" (in what is a new example of synesthesia), "it is whiteness that drains", "white light that melts", and, finally, "the fish that spills on the silver". Now, the colors are languishing, and they become deliberately too imprecise to reproduce Matisse's work faithfully. The same happens in the poem "Looking at a Painting by Jansen", where the blurred style of the French painter moves in tandem with the poem: "A world, all slow, / that melts / and strips, silently". Agelet is 
therefore an exceptional case amongst poets who have become painters, because he transforms his poems into paintings and thereby reveals the constructive mechanisms of both arts.

\section{Conclusion}

This article has verified how the reading and dissemination of illustrated albums has extended the traditional concept of reading to reading images and illustrations. Young people have multiple ways of approaching the art world through reading. The illustrated album has become an essential instrument in discovering and deepening concepts and characteristics of the pictorial world. The article has also shown us how to read about painting, and especially how to read poetry that reproduces the painting, revealing to the reader or the spectator the mechanisms of the reading process.

Reading about art allows us to understand more clearly a reality that identifies us as humans, as sensitive beings (Eisner, 2004), and it gives us knowledge that allows us to enjoy a painting and experience aesthetic pleasure, which then makes it possible to develop imagination and expressiveness. In this sense, works such as those analyzed make it easy for us to learn about art, one of the most genuinely human forms of expression.

In addition, reading texts that reproduce the qualities of painting enables us to verify how rhythm, verse, rhyme, literary resources, the denotative use of language, and the ability to associate diverse realities serve to express the emotion that occurs in a painting. Reading ecphrastic painting is to learn to read literature because its creative mechanisms are based precisely on the use of the resources that identify and individualize literary language, that resemble painting and turn it into art. Reading poetry about painting is to learn to interpret poetry and its mechanisms, and reading expository texts about painting is to learn to contemplate painting, to appreciate its intrinsic characteristics. As Horace stated, "Ut pictura poesis" [as is painting so is poetry], because to read is to broaden the reader's horizon of expectations; it is to learn; it is ultimately to be a better human being.

\section{References}

Agelet i Garriga, J (2008). Tria de versos. Lleida: Publicaciones de la Universitat de Lleida.

Arañó Gisbert, J.C. (1994). Arte, educación y creatividad. Pixel-Bit: Revista de medios y educación, 2, 65-87.

Bamford, A. (2009). El factor ;Wuau! El papel de las artes en la educación. Un estudio internacional sobre el impacto de las artes en la educación. Barcelona: Octaedro.

Bosch, E. (2007) Hacia una definición de álbum. Anuario de Investigación en literatura infantil y juvenil, 5, 25-46.

Cansino, E (1998). El misterio Velázquez. Madrid: Bruño.

Clar, R., Llaurado, A., Riera, A., Quinquer, D., \& Roca, M. (2007) Hablar, leer y escribir para aprender desde las áreas curriculares. Aula de Innovación Educativa, 159, 7-9

Deleuze, G., \& Guattari, F. (1987). Mil mesetas, capitalismo y esquizofrenia. Valencia: PreTextos.

Duran, T. (2010). L'àlbum dins la narrativa visual. Articles de didàctica de la llengua i literatura, $52,10-22$. 
Eisner, E. (2002) El arte y la creación de la mente. Barcelona: Paidós.

Eisner, E. (2004) El Arte y la Creación de la Mente: El Papel de Las Artes Visuales en la Transformación de la Conciencia. Madrid: Paidos

Falguera, E. (2010) La pintura d'un poeta, Actes del XVè Col·loqui Internacional de Llengua i Literatura Catalanes. Barcelona: PAM.

Laneyrie-Dagen, N. (2013). Leer la pintura. Barcelona: Larousse.

Lessing, G. E. (2014). Laocoonte o sobre los límites de la pintura y la poesía. Barcelona: Herder Editorial.

López-Rodríguez, F. (2007) (ed.). La educación artística en la escuela. Barcelona: Graó.

Martín-Gil F. (2011). Claves para entender un cuadro. Madrid: AIIM

Mendívil Trelles de Peña, L. (2011). El arte en la educación de la primera infancia: una necesidad impostergable. Educación, Vol. XX, 39, 23-36

Morin, E. (1999). Los siete saberes necesarios para la educación del futuro. México: Unesco.

Renshaw, A. (2006). El abc del arte para niños. Barcelona: Phaidon.

Rosenstock, B. (2015). El sonido de los colores. Barcelona: Juventud.

Solé, I. (1992). Estrategias de lectura. Barcelona: Graó.

Tabernero, R. (2013). Claves para una poética de la recepción del libro-álbum: Un lector inserto en una "inmensa minoría. [online]. IBBY. http://www.ibby.org/index.php?id=1062

Tabernero, R. (2012). La hipertextualidad como fundamento de construcción en la literatura "iluminada": la formación del lector en el libro-álbum y en el libro ilustrado. Leer Hipertexto. Del marco hipertextual a la formación de lector literario. Barcelona: Octaedro.

Tabernero, R. (2009). Leer mirando. El libro-álbum en la promoción de hábitos lectores. Claves para una poética de su lectura. I Encuentro Internacional de Estudio y Debate. Literatura Infantil y Matices. p. 9-44.

Vasquez, C. (2010). El arte un derecho humano. Recovered from http://www.chalenavasquez.com/pdf/El_arte, un derecho humano.pdf

Vicente-Yagüe, M. ${ }^{\mathrm{a}}$ I. (2016). Artes y literatura en la LOMCE: mecanismos para la comprensión de lecturas plurales. Investigaciones sobre lectura, 5, 59-69.

Winter, J (2013). Las tijeras de Matisse. Barcelona: Juventud. 